\title{
Computationally Efficient Graph Matching via Energy Vector Extraction
}

\begin{abstract}
This paper presents a method for graph matching based on domain knowledge by quantifying representative graph features. Our method searches and extracts the most relevant cues in different graphs. Once these cues are extracted and quantified, a new energy function is used to match the different graphs based on the obtained features values. This approach has been successfully applied for deformable template matching. As a result the error of matching is reduced, as well as the computational cost by efficiently selecting and grouping representative features.
\end{abstract}

\section{Introduction}

Graphs hold a great representative power which makes them the most natural way to encode and symbolize any pattern [1]. All non-verbal human communications, ranging from hand gestures to written documents, include some concept of a graph. Many applications in computer vision include selecting and extracting information from graphs, since graphs provide a useful way to represent knowledge. For this reason Tombre proposed a method for analyizing engineering drawings [2], and Kruger, Potzsch, and Andmalsburg proposed a method for determining face position and pose based on labeled graphs [3].

Due to this power, graph matching is a logical way to compare and recognize different objects. The main challenge in any graph matching method is to extract representative segments of different graphs for comparison. Many approaches have been considered: some were based on one-to-one correspondence [1], while others were based on many-to-many correspondence [4]. However, these methods usually suffered from computational complexity and inefficiency while handling distortions. In this paper, we present a method for graph matching based on feature extraction to obtain a vector representation for each graph which is applied for deformable template recognition. A new energy function is formulated to extract this vector based on the graph attributes. Then, a simple distance function is applied for the final 
matching. This method is robust to noise, segmentation errors, graphs with missing or extra edges, and graphs with missing or extra node.

This paper is organized as follows. Section 2 discusses briefly related works. Section 3 presents the feature extraction method while section 4 presents the matching method. In section 5, experimental results are discussed. Concluding remarks are available in section 6 .

\section{Related Work}

Graph matching is usually addressed as a method for object recognition. Gold and Rangarajan [1] propose a one-to-one correspondence is established through a graduated assignment Kim and Kak [5] propose a bipartite graph matching using discrete relaxation for 3-D object reconstruction. Other algorithm uses a quadratic programming approach for graph matching using a maximal clique framework [6]. Many-tomany correspondence has been also investigated [4], frequently in the context of edit-distance, [7].

However, these methods are either computationally complex or does not support slight distortions in graphs. So, a small deformation in the graph to be matched introduces a lot of error in the matching result, unless the algorithm is computationally expensive.

In this paper, a robust and low computational cost method is introduced. Its strength lies in its ability to assign quantitative values to representative graph features such that the matching process is only a simple distance function.

\section{Energy Function}

A graph $G=(N, E)$ can be interpreted as a set of basic forms which are nodes and edges. Figure 1 shows an interpretation of a given object as a graph. The features used in matching different graphs are the information obtained from the nodes and edges.

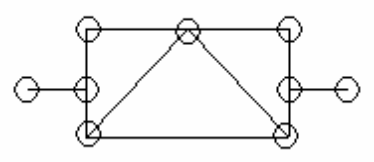

Fig. 1. Object representation as a set of nodes and edges

This method transforms the graph into a vector of energy values. An energy function is calculated for each node in the graph. This energy function aims at encoding the number of edges connected to each node, their relative length, their shape, and the average relative angle of each node. Then, each graph is represented as a vector of energy values calculated at each of its nodes. 
Towards this end, we define a novel energy function as follows:

$\mathrm{E}(i)=\sum_{p=1}^{n} p * k 1+\frac{\sum_{p=1}^{n} L_{i p}}{\sum_{j=1}^{m} \sum_{p=1}^{n} L_{j p}} * k 2+\frac{\sum_{p=1}^{n} \theta_{i p}}{n} * \frac{k 3}{2 . \pi}+\frac{\sum_{p=1}^{n} A_{i p}}{\sum_{j=1}^{m} \sum_{p=1}^{n} A_{j p}} * k 4$

where $n$ is the number of edges attached to node $i, m$ is the number of nodes in the graph, $L_{i p}$ is the length of the pth edge attached to node $i, \theta_{i p}$ is the angle the pth edge form with the horizontal, $A_{i p}$ is the area enclosed by the edge in case it is not a straight line. This area is representative of the shape of the edge as shown in Fig. 2.

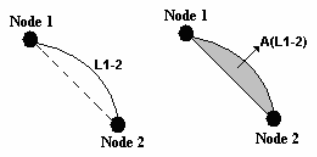

Fig. 2. Area enclosed by a circular edge between Node 1 and Node 2

Lastly, k1, k2, k3, and k4 are weights based on the characteristics of the considered graphs.

\section{Graph Matching}

Let's assume a graph $\mathbf{G}_{\mathbf{k}}$ is to be matched with a set of graphs $\mathbf{G}_{\mathbf{1}}, \mathbf{G}_{\mathbf{2}}, \ldots, \mathbf{G}_{\mathbf{n}}$. Once the energy vectors $\mathbf{E}_{\mathbf{k}}$ and $\mathbf{E}_{\mathbf{1}}, \mathbf{E}_{2}, \ldots, \mathbf{E}_{\mathbf{n}}$ are obtained, they are then sorted in descending order. Then, the distance between $\mathbf{E}_{\mathbf{k}}$ and each of the other energy vectors is calculated according to Eq. 2.

$$
d_{j}=\sum_{i=1}^{n}\left|E_{k}(i)-E_{j}(i)\right|
$$

where $d_{j}$ is the distance between $G_{k}$ and $G_{j}, n$ is the maximum number of nodes found in $G_{k}$ and $G_{j}$.

\subsection{Robustness}

This method is robust to perturbations in graph structure due to the fact that the energy function, Eq. 1, emphasizes the similarities between graphs. The idea is to compare the most representative features of one graph to the most representative features of the second graph. Therefore, sorting the two energy vector, and comparing them one-by-one (the first value in $\mathbf{E}_{1}$ to the first value in $\mathbf{E}_{2}$, the second value in $\mathbf{E}_{1}$ to the second value of $\mathbf{E}_{2}$, and so on) provide a very simple and effective way to compare the representative features. Any noise or distortion introduced will have low energy value and will appear at the end of the energy vector; therefore, it will have a minimal effect on the matching process. Also, this matching process is independent on the size of the graphs, since the relative lengths of the edges are considered. Matching is also independent on the position of the graphs since relative angles are considered. 


\subsection{Efficiency}

The main advantage of this method is its computational efficiency. It is able to achieve robust detection in $\mathrm{O}(\mathrm{n})$ where $n$ is the maximum number of nodes found in the different graphs. This is considered a great achievement when compared with other methods that are considered low-cost yet they run in $\mathrm{O}(\mathrm{lm})$, where $l$ and $m$ are the number of links in the two graphs [1].

\section{Experimental Results}

We worked with a database ${ }^{1}$ of four graphs and 500 distorted versions of these graphs. The four graphs are shown in Fig. 3 and their corresponding distorted versions are shown in Fig. 4.

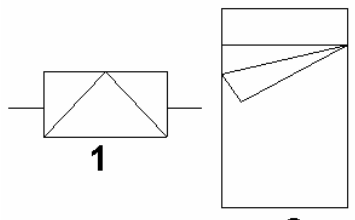

2

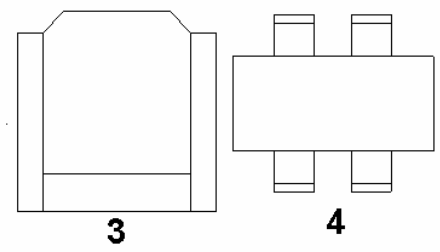

Fig. 3. The four basic graphs

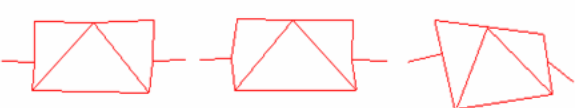

(a)

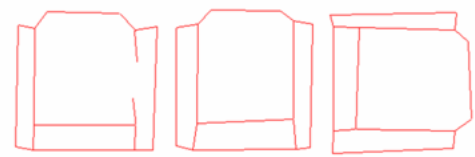

(c)

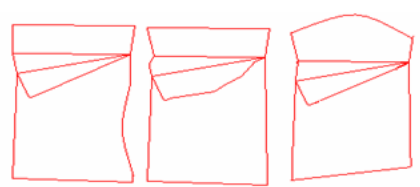

(b)
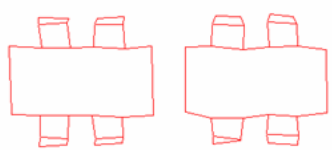

(d)

Fig. 4. (a) Distorted versions of Graph 1 (b) Distorted versions of Graph 2 (c) Distorted versions of Graph 3 (d) Distorted versions of Graph 4

${ }^{1}$ International Symbol Recognition Contest at GREC 2003, Barcelona, Catalonia, Spain, July 29, 2003 
The purpose was to match each distorted graph with its original version. Setting experimentally $\mathrm{k} 1$ to $10, \mathrm{k} 2$ to $5, \mathrm{k} 3$ to 3 , and $\mathrm{k} 4$ to 3 , we were able to achieve a $100 \%$ recognition rate. Table 1 shows the result of calculating the distance function between 16 distorted versions of Graph 1 and all four graphs. It can be easily seen that the minimum distance is produced when comparing each one (on each row) with Graph 1 indicating that the matching was done correctly. Tables 2, 3, and 4 show the same results for Graphs 2, 3, and 4 respectively.

TABLE 1

Distance Calculation for 16 Distorted Versions of Graph 1

\begin{tabular}{|c|c|c|c|}
\hline$(1-(a))$ & $(2-(a))$ & $(3-(a))$ & $(4-(a))$ \\
\hline 9 & 106 & 157 & 663 \\
\hline 12 & 107 & 162 & 670 \\
\hline 12 & 103 & 158 & 666 \\
\hline 8 & 101 & 154 & 662 \\
\hline 10 & 103 & 154 & 671 \\
\hline 11 & 106 & 154 & 668 \\
\hline 11 & 107 & 162 & 670 \\
\hline 9 & 108 & 159 & 668 \\
\hline 10 & 110 & 160 & 669 \\
\hline 12 & 110 & 160 & 664 \\
\hline 8 & 112 & 158 & 665 \\
\hline 8 & 104 & 154 & 670 \\
\hline 12 & 106 & 169 & 669 \\
\hline 11 & 111 & 154 & 663 \\
\hline 10 & 106 & 153 & 667 \\
\hline 9 & 104 & 161 & 670 \\
\hline
\end{tabular}

TABLE 2

Distance Calculation for 16 Distorted Versions of Graph 2

\begin{tabular}{|c|c|c|c|}
\hline (1-(b)) & (2-(b)) & (3-(b)) & (4-(b)) \\
\hline 94 & 8 & 172 & 660 \\
\hline 96 & 12 & 176 & 664 \\
\hline 94 & 8 & 172 & 660 \\
\hline 96 & 12 & 176 & 664 \\
\hline 96 & 12 & 176 & 663 \\
\hline 94 & 8 & 172 & 660 \\
\hline 96 & 9 & 172 & 660 \\
\hline 94 & 8 & 176 & 663 \\
\hline 97 & 11 & 174 & 665 \\
\hline 97 & 10 & 174 & 663 \\
\hline 95 & 11 & 175 & 660 \\
\hline 94 & 8 & 176 & 662 \\
\hline 95 & 9 & 175 & 664 \\
\hline 96 & 10 & 173 & 660 \\
\hline 94 & 8 & 175 & 664 \\
\hline 94 & 8 & 174 & 663 \\
\hline
\end{tabular}


TABLE 3

Distance Calculation for 16 Distorted Versions of Graph 3

\begin{tabular}{|c|c|c|c|}
\hline (1-(c)) & (2-(c)) & (3-(c)) & (4-(c)) \\
\hline 158 & 177 & 8 & 572 \\
\hline 155 & 178 & 4 & 567 \\
\hline 155 & 176 & 5 & 571 \\
\hline 157 & 180 & 3 & 569 \\
\hline 155 & 176 & 5 & 571 \\
\hline 157 & 180 & 8 & 569 \\
\hline 158 & 180 & 7 & 567 \\
\hline 155 & 176 & 3 & 572 \\
\hline 156 & 178 & 4 & 571 \\
\hline 154 & 177 & 6 & 569 \\
\hline 155 & 176 & 7 & 571 \\
\hline 158 & 180 & 8 & 570 \\
\hline 156 & 177 & 7 & 571 \\
\hline 156 & 180 & 3 & 568 \\
\hline 158 & 177 & 3 & 572 \\
\hline 157 & 176 & 5 & 576 \\
\hline
\end{tabular}

TABLE 4

Distance Calculation for 16 Distorted Versions of Graph 4

\begin{tabular}{|c|c|c|c|}
\hline$(1-(d))$ & $(2-(d))$ & $(3-(d))$ & $(4-(d))$ \\
\hline 667 & 676 & 571 & 33 \\
\hline 667 & 686 & 577 & 31 \\
\hline 669 & 678 & 569 & 23 \\
\hline 670 & 679 & 570 & 24 \\
\hline 670 & 679 & 570 & 28 \\
\hline 670 & 675 & 568 & 24 \\
\hline 669 & 678 & 571 & 17 \\
\hline 668 & 678 & 570 & 22 \\
\hline 671 & 679 & 569 & 25 \\
\hline 669 & 675 & 568 & 29 \\
\hline 670 & 676 & 573 & 23 \\
\hline 667 & 682 & 567 & 19 \\
\hline 670 & 680 & 568 & 28 \\
\hline 669 & 679 & 572 & 30 \\
\hline 671 & 677 & 570 & 26 \\
\hline & & 569 & 25 \\
\hline
\end{tabular}

\section{Conclusions}

In this paper, we present a computationally efficient graph matching technique based on feature extraction via energy vectors. This method has a very low order computational complexity $\mathrm{O}(\mathrm{n})$ and it is extremely robust to noise and distortion. The experimental results were impressive. In future, this method will be tested with more graphs specifically those including non-linear edges. 


\section{References}

[1] Gold, S. and Rangarajan, A., "Graph Matching by Graduated Assignment”, IEEE Computer Society Conference on Computer Vision and Pattern Recognition(VCPR'96), pp. 239-244, June 1996. San Francisco, CA, USA.

[2] Tombre, K., “Analysis of Engineering Drawings: State of the Art and Challenges”, Graphics Recognition Algorithms and Systems, LNCS, vol. 1389, pp. 257-264, 1998.

[3] Kruger, N., Potzsch, M., Andmalsburg, C.V.D., "Determination of Face Position and Pose with a Learned Representation Based on Labeled Graphs”, Image Vision Computing, n. 15, pp. 665-673, 1997.

[4] Keselman, Y., Shokoufandeh, A., Demirci, M.F. and Dickinson, S., "Many-to-Many Graph Matching via Metric Embedding”, IEEE Computer Society Conference on Computer Vision and Pattern Recognition, vol. 1, pp. 1-850 - 1-857, June 2003.

[5] Kim, W., and Kak, A.C., "3D object recognition using bipartite matching embedded in discrete relaxation”, IEEE Transactions on Pattern Analysis and Machine Intelligence, 13(3):224-251, 1991.

[6] Pelillo, M., Siddiqi, K., and Zucker, S., "Matching hierarchical structures using association graphs”, IEEE Transactions on Pattern Analysis and Machine Intelligence, 21(11):11051120, November 1999.

[7] Liu, T.L., and Geiger, D., “Approximate tree matching and shape similarity”, $7^{\text {th }}$ International Conference on Computer Vision, pp. 456-462, 1999. 